\title{
Electrolytes and clinical outcomes in patients with acute ischemic stroke or transient ischemic attack
}

\author{
Anxin Wang ${ }^{1,2}$, Xue Tian ${ }^{3,4}$, Hongqiu Gu ${ }^{1,2}$, Yingting Zuo ${ }^{3,4}$, Xia Meng ${ }^{1,2}$, Pan Chen ${ }^{1,2}$, Hao Li ${ }^{1,2}$, \\ Yongjun Wang ${ }^{1,2}$
}

${ }^{1}$ China National Clinical Research Center for Neurological Diseases, Beijing Tiantan Hospital, Capital Medical University, Beijing, China; ${ }^{2}$ Department of Neurology, Beijing Tiantan Hospital, Capital Medical University, Beijing, China; ${ }^{3}$ Department of Epidemiology and Health Statistics, School of Public Health, Capital Medical University, Beijing, China; ${ }^{4}$ Beijing Municipal Key Laboratory of Clinical Epidemiology, Beijing, China

Contributions: (I) Conception and design: Y Wang, A Wang; (II) Administrative support: Y Wang; (III) Provision of study materials or patients: X Meng, P Chen, H Li, Y Wang; (IV) Collection and assembly of data: A Wang, H Gu; (V) Data analysis and interpretation: A Wang, X Tian, Y Zuo; (VI) Manuscript writing: All authors; (VII) Final approval of manuscript: All authors.

Correspondence to: Yongjun Wang, MD. China National Clinical Research Center for Neurological Diseases, Beijing Tiantan Hospital, Capital Medical University, No. 119 South 4th Ring West Road, Fengtai District, Beijing 100070, China. Email: yongjunwang@ncrcnd.org.cn.

\begin{abstract}
Background: Abnormal electrolytes were closely related to the prognosis of various diseases, the prognostic role of electrolytes in stroke has not been investigated well. We aimed to investigate the association between electrolytes and clinical outcomes in patients with acute ischemic stroke (AIS) or transient ischemic attack (TIA).

Methods: Data were recruited from the China National Stroke Registry III study. Patients were classified into three groups according to tertiles and the normal range of each electrolyte. Multivariable logistic and Cox proportional hazards regressions were adopted to explore the associations of electrolytes with poor functional outcomes [modified Rankin Scale (mRS) 3-6/2-6] and all-cause death at 3 months and 1 year.

Results: A total of 10,299 eligible patients were enrolled. After adjusted for confounding factors, the first tertile electrolytes were associated with increased risk of poor functional outcome (mRS score 3-6) at 1 year, the adjusted odds ratios (95\% confidence intervals) were 1.33 (1.14-1.55) for potassium, 1.41 (1.20-1.60) for sodium, 1.27 (1.08-1.48) for chloride, compared with the second tertile. Similar results were found when poor functional outcome was defined as mRS score 2-6 and all-cause death. However, almost no significant association was present of calcium with these outcomes. All results were consistent when each electrolyte was classified into three groups according to the normal range and the outcomes timepoint was set at 3 months.

Conclusions: Lower levels of potassium, sodium, chloride but not calcium were associated with higher risk of poor functional outcomes and death in patients with AIS or TIA.
\end{abstract}

Keywords: Electrolytes; acute ischemic stroke (AIS); transient ischemic attack (TIA); clinical outcomes

Submitted Feb 15, 2021. Accepted for publication Apr 02, 2021.

doi: $10.21037 / \mathrm{atm}-21-741$

View this article at: http://dx.doi.org/10.21037/atm-21-741

\section{Introduction}

Ischemic stroke and transient ischemic attack (TIA) are common cerebrovascular diseases and associated with high disability and mortality rates (1). The world health organization has reported that approximately 15 million people suffer a stroke annually, of which 5 million are fatal and 5 million result in permanent disability (2). Identifying and controlling stroke-related risk factors would be extremely important in the treatment and secondary prevention of stroke.

Electrolyte disorders as a complication of dehydration, 
diabetes, renal failure, or myocardial infraction are frequently observed in clinical practice (3-5). Hypokalemia, hyponatremia, hypochloremia, and hypocalcemia were common electrolyte abnormalities encountered in clinical practice. Previous studies have demonstrated that these electrolyte disorders were also closely related to the prognosis of heart failure (6), acute coronary syndrome (7), chronic kidney disease (8), hypertension (9) intracerebral hemorrhage (8), and acute pancreatitis (10). Although some studies have investigated the prognosis role of hypokalemia, hyponatremia, and hypochloremia in stroke, the sample size was small and the results were conflicting (11-17). Furthermore, limited data from large multicenter prospective studies were available to evaluate the role of electrolytes in the prognosis of stroke, and the associations of each electrolyte category with short- and long-term outcomes in patients with stroke have not been investigate well.

Therefore, in the present study, we aimed to investigate the associations of electrolytes (potassium, sodium, chloride, and calcium) with poor functional outcomes and all-cause death among patients with acute ischemic stroke (AIS) or TIA at the time point of 3 months and 1 year, using data from the multicenter cohort of the Third China National Stroke Registry (CNSR-III). We present the following article in accordance with the STROBE reporting checklist (available at http://dx.doi.org/10.21037/atm-21-741).

\section{Methods}

\section{Study population}

Data were derived from the CNSR-III, which is a nationwide prospective registry for patients presented to hospitals with acute ischemic cerebrovascular events. The registry recruited patients consecutively from 201 hospitals of 22 provinces and four municipalities between August 2015 and March 2018 in China. Finally, 15,166 patients with AIS or TIA within 7 days from the onset of symptoms were enrolled. The design of the CNSR-III has been described in detail previously (18). The CNSR-III was approved by ethics committees of Beijing Tiantan Hospital and all participating centers. The study was conducted in accordance with the Declaration of Helsinki (as revised in 2013). All participants or their legal proxies have provided written informed consent. The study was approved by the ethics committee of Beijing Tiantan Hospital (No.: KY2015-001-01) and all study centers gave ethical approval of the study protocol.

\section{Baseline data collection}

Baseline data were collected through a direct interview or medical records, including age, sex, body mass index (BMI, $\mathrm{kg} / \mathrm{m}^{2}$ ), medical history of hypertension, diabetes mellitus, dyslipidemia, stroke or TIA, atrial fibrillation or flutter, peripheral vascular disease, heart failure; stroke types (IS or TIA), the etiology classification of ischemic stroke performed according to the TOAST (Trial of Org 10172 in Acute Stroke Treatment) criteria (19), smoking status, medication use (including using of cholesterollowering, antihypertensive, antidiabetic, antiplatelet, and anticoagulant agents); National Institutes of Health Stroke Scale (NIHSS); time from onset of symptoms to admission, and laboratory test of plasm lipids, fasting blood glucose (FBG), estimated glomerular filtration rate (eGFR), and high-sensitivity C-reactive protein (hs-CRP).

\section{Electrolyte testing and definition of electrolyte abnormalities}

Fasting serum and plasma specimens were extracted and transported through cold chain to the core laboratory in Beijing Tiantan Hospital. All specimens were stored at -80 ${ }^{\circ} \mathrm{C}$ until detected centrally and blindly. Concentrations of serum electrolytes were analyzed by automated hematology analyzer. All measurements were performed by laboratory personnel who were blinded to patients' clinical situations.

\section{Follow-up and outcome evaluation}

Participants were followed-up via face-to-face or telephone interview at for clinical outcomes at 3 months and 1 year by trained research personnel. Poor functional outcome was defined as modified Rankin Scale (mRS) score ranged $3-6 / 2-6$. All-cause death was defined as death from any cause and was confirmed on a death certification from the participating hospital or the local citizen registry.

\section{Statistical analysis}

Continuous variables were presented as medians and interquartile range because of skewed distribution. Categorical variables were presented as frequencies and percentages. The nonparametric Wilcoxon or KruskalWallis test were used to compare group differences for 
continuous variables, and chi-square tests were used for categorical variables.

In the main analyses, participants were divided into three groups by electrolyte tertiles for each electrolyte category, and the second tertile was defined as the reference group. The associations of electrolytes with poor functional outcomes and all-cause death were investigated with logistic regression and Cox proportional hazard models, respectively. Unadjusted and adjusted models were constructed, the adjusted model was adjusted for all variables at baseline. The robust sandwich estimator for the covariance matrix was used in the models accounting for the clustering effect at the hospital level. In addition, to capture the dose-response relationship, the association between electrolytes and the outcomes on a continuous scale was examined using restricted cubic splines, with 3 knots at the 10th, 50th, 90th percentiles of each electrolytes distribution (20). Reference points for serum potassium, sodium, chloride, and calcium were the median (3.93, 141, 104 , and $2.25 \mathrm{mmol} / \mathrm{L}$, respectively) of the reference group (the second tertile), and odds ratio (OR)/hazard ratio (HR) was adjusted for all confounding variables. Furthermore, ordinal logistic regression was applied to estimate the common odds ratio for a shift in the direction of a worse outcome on the mRS score at both 3 months and 1 year according to each electrolyte category.

In the secondary analysis, we aimed to determine the association of hypoelectrolytemia and hyperelectrolytemia with these outcomes. The participants were classified into 3 groups according to normal range of electrolytes, and those with normal range were taken as the reference. The reference range of serum potassium, sodium, chloride, and calcium were $3.5-4.7 \mathrm{mmol} / \mathrm{L}$ (21), 135-145 $\mathrm{mmol} / \mathrm{L}$ (21), 98-109 mmol/L (16,22), and 2.25-2.58 mmol/L (21), respectively. Values less than the lower range were considered as hypoelectrolytemia, and values greater than the upper range as hyperelectrolytemia.

Statistical analyses were performed with SAS software version 9.4 (SAS Institute Inc., Cary, NC, USA). All statistical tests were 2-sided and the significance level was set at 0.05 .

\section{Results}

\section{Baseline characteristics}

We excluded 4,533 patients without available data on electrolytes, and 334 patients with missing available mRS at the time point of 3 months or 1 year, therefore, a total of 10,299 patients were eligible for the current analysis. The included patients had less men, were more likely to have a history of dyslipidemia, higher total cholesterol, highdensity lipoprotein cholesterol, FBG and hs-CRP level, and a lower proportion of IS, large-artery atherosclerosis, cholesterol-lipid agents, lower eGFR, sodium and chloride levels (Table S1 in the online-only data supplement).

Baseline characteristics of the patients stratified by survival status are presented in Table 1 . The median age of the included patients was 63 years, and 6,980 (67.77\%) were men. The median (interquartile range) of potassium, sodium, chloride, and calcium were 3.93 (3.68-4.19), 141.00 (139.00-142.70), 104.00 (101.70-1.06.10), and 2.25 (2.17-2.34) $\mathrm{mmol} / \mathrm{L}$, respectively. The survivors were younger, had more men, higher BMI, more current smokers, antiplatelet agents takers, a higher proportion of NIHSS $\leq 15$, higher TG, eGFR, sodium, chloride, and calcium levels; and were less likely to have a history of stroke, arterial fibrillation/flutter, heart failure, a lower proportion of IS, large-artery atherosclerosis, anticoagulant agents, lower baseline NIHSS score, FBG and hs-CRP levels than non-survivors. Moreover, compared with patients without poor functional outcomes (mRS score $<3$ or 2), those with poor functional outcomes had the similar pattern of baseline characteristic (Table S2 in the onlineonly data supplement).

\section{Association of electrolytes with poor functional outcomes}

Poor functional outcome (mRS score 3-6) occurred in 1,477 (14.34\%) patients and mRS score 2-6 occurred in 2,787 $(27.06 \%)$ patients at 3 months, while at 1-year follow-up, $1,396(13.55 \%)$ patients had mRS score 3-6, 2,503 (24.30) patients had mRS score 2-6.

The association between electrolytes and poor functional outcomes are presented as Table 2 and Figure 1. At the time point of 1 year, after adjusted for potential covariates, electrolytes in the first tertile group were associated with increased risk of poor functional outcome (mRS score 3-6), the adjusted ORs with 95\% CIs were 1.33 (1.14-1.55) for potassium, $1.41(1.20-1.60)$ for sodium, and 1.27 (1.08-1.48) for chloride, compared with the second tertile of electrolytes. Multivariable-adjusted spline regression models showed L-shaped associations of potassium, sodium, and chloride with the risk of poor functional outcome (mRS score 3-6) (Figure 2A,B,C). Furthermore, there were significant shifts in the distribution of the mRS scores 
Table 1 Baseline characteristics of patients according to survival status at 1 year

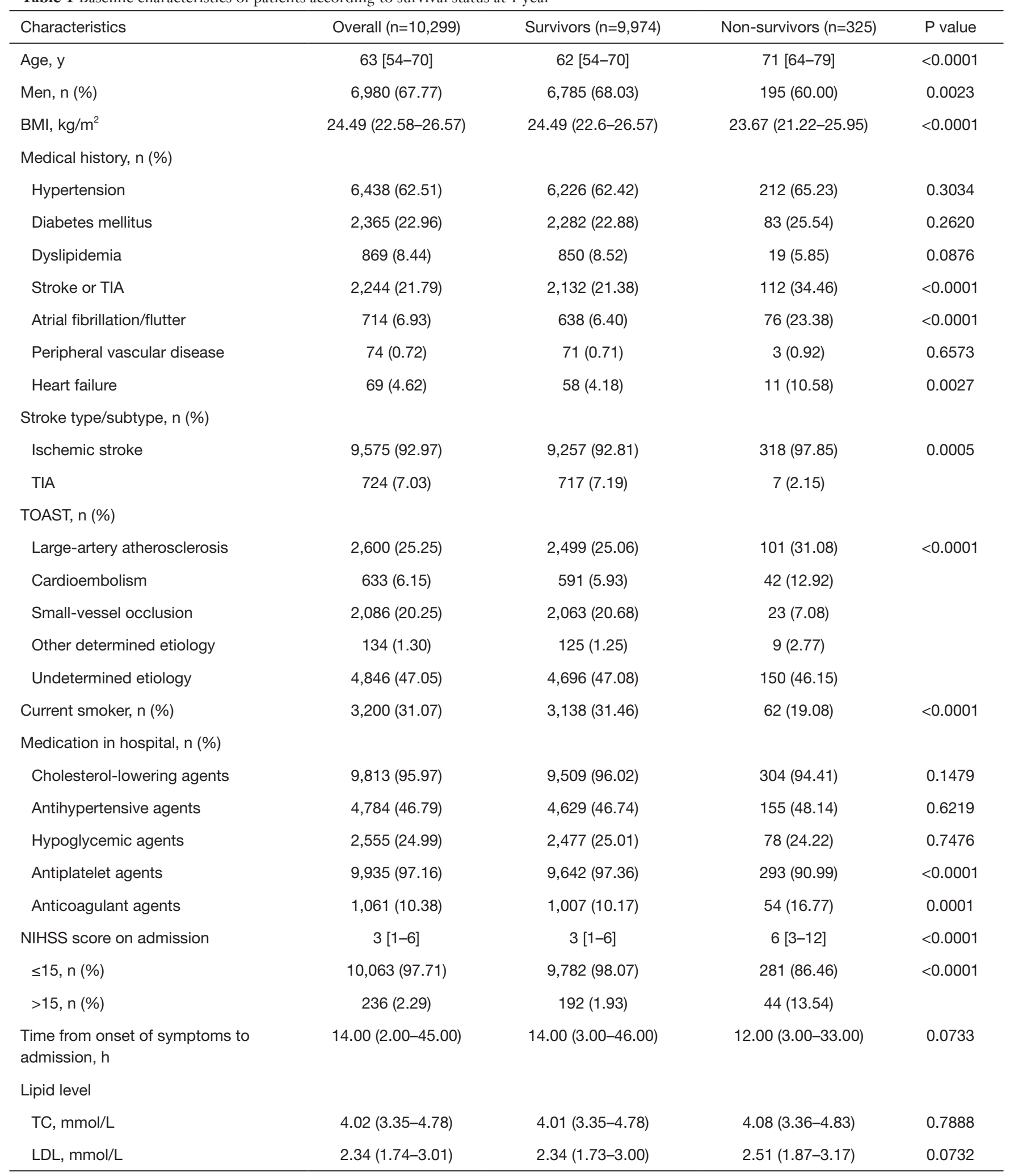

Table 1 (continued) 
Table 1 (continued)

\begin{tabular}{|c|c|c|c|c|}
\hline Characteristics & Overall $(n=10,299)$ & Survivors $(n=9,974)$ & Non-survivors $(n=325)$ & $P$ value \\
\hline $\mathrm{TG}, \mathrm{mmol} / \mathrm{L}$ & $1.37(1.03-1.87)$ & $1.37(1.03-1.88)$ & $1.20(0.90-1.64)$ & 0.0001 \\
\hline $\mathrm{FBG}, \mathrm{mmol} / \mathrm{L}$ & $5.56(4.90-6.91)$ & $5.55(4.90-6.90)$ & $6.01(5.06-7.78)$ & 0.0023 \\
\hline eGFR, $\mathrm{mL} / \mathrm{min} / 1.73 \mathrm{~m}^{2}$ & $92.93(80.85-101.84)$ & 93.17 (81.38-102.10) & 83.09 (65.99-92.99) & $<0.0001$ \\
\hline Potassium, $\mathrm{mmol} / \mathrm{L}$ & $3.93(3.68-4.19)$ & $3.93(3.69-4.19)$ & $3.90(3.60-4.26)$ & 0.5750 \\
\hline Sodium, mmol/L & $141.00(139.00-142.70)$ & $141.00(139.00-142.70)$ & $140.00(137.50-142.40)$ & 0.0005 \\
\hline Chloride, $\mathrm{mmol} / \mathrm{L}$ & $104.00(101.70-1.06 .10)$ & $104.00(101.70-106.10)$ & $103.20(100.31-106.00)$ & 0.0026 \\
\hline Calcium, mmol/L & $2.25(2.17-2.34)$ & $2.25(2.17-2.34)$ & $2.23(2.14-2.33)$ & 0.0047 \\
\hline
\end{tabular}

Continuous variables are expressed as median with interquartile range. Categorical variables are expressed as frequency with percentage. BMI, body mass index; TIA, transient ischemic Attack; TOAST, Trial of Org 10172 in Acute Stroke Treatment; NIHSS, The National Institutes of Health Stroke Scale; TC, total cholesterol; LDL, low-density lipoprotein cholesterol; HDL, high-density lipoprotein cholesterol; TG, triglycerides; FBG, fasting blood glucose; eGFR, estimated glomerular filtration rate; hs-CRP, high sensitivity C-reactive protein.

Table 2 Adjusted odds ratio (95\% confidence interval) for poor functional outcome (mRS score 3-6) according to tertiles of serum electrolyte indicators

\begin{tabular}{|c|c|c|c|c|c|c|c|}
\hline \multirow{2}{*}{$\begin{array}{l}\text { Electrolyte } \\
\text { indicator }\end{array}$} & \multirow{2}{*}{ Tertiles } & \multicolumn{3}{|c|}{ mRS score $3-6$ at 3 months } & \multicolumn{3}{|c|}{ mRS score $3-6$ at 1 year } \\
\hline & & Events, $\mathrm{n}(\%)$ & Unadjusted & Adjusted $^{\dagger}$ & Events, n (\%) & Unadjusted & Adjusted $^{\dagger}$ \\
\hline \multirow{3}{*}{$\begin{array}{l}\text { Potassium, } \\
\mathrm{mmol} / \mathrm{L}\end{array}$} & $\mathrm{T} 1(<3.78)$ & $592(17.28)$ & 1.38 (1.20-1.59) & $1.20(1.12-1.51)$ & $556(16.23)$ & 1.39 (1.21-1.61) & $1.33(1.14-1.55)$ \\
\hline & T2 (3.78-4.09) & $432(12.85)$ & Reference & Reference & 406 (12.08) & Reference & Reference \\
\hline & T3 (>4.09) & $453(12.90)$ & $1.06(0.91-1.23)$ & $1.07(0.91-1.25)$ & $434(12.36)$ & $1.09(0.94-1.27)$ & $1.09(0.92-1.28)$ \\
\hline \multirow{2}{*}{$\begin{array}{l}\text { Sodium, } \\
\mathrm{mmol} / \mathrm{L}\end{array}$} & T2 (139.70-141.90) & $398(12.90)$ & Reference & Reference & $361(11.70)$ & Reference & Reference \\
\hline & T3 $(>141.90)$ & $481(12.70)$ & $1.00(0.86-1.16)$ & $1.01(0.86-1.19)$ & $474(12.51)$ & $1.13(0.97-1.32)$ & $1.13(0.96-1.33)$ \\
\hline \multirow{2}{*}{$\begin{array}{l}\text { Chloride, } \\
\mathrm{mmol} / \mathrm{L}\end{array}$} & T1 $(<102.50)$ & $578(16.91)$ & $1.40(1.21-1.61)$ & $1.30(1.11-1.52)$ & $534(15.62)$ & $1.35(1.17-1.56)$ & $1.27(1.08-1.48)$ \\
\hline & T2 (102.50-105.47) & 445 (13.02) & Reference & Reference & $424(12.40)$ & Reference & Reference \\
\hline \multirow{2}{*}{$\begin{array}{l}\text { Calcium, } \\
\mathrm{mmol} / \mathrm{L}\end{array}$} & T2 (2.20-2.30) & $497(14.19)$ & Reference & Reference & $476(13.59)$ & Reference & Reference \\
\hline & T3 $(>2.30)$ & $454(13.36)$ & $0.95(0.82-1.10)$ & $1.00(0.85-1.16)$ & $393(11.57)$ & $0.81(0.70-0.94)$ & $0.85(0.72-1.00)$ \\
\hline
\end{tabular}

\footnotetext{
${ }^{\dagger}$, adjusted for age, gender, body mass index, history of hypertension, diabetes mellitus, dyslipidemia, stroke or TIA, atrial fibrillation/ flutter, peripheral vascular disease, heart failure, stroke type, TOAST, current smoke, cholesterol-lowering agents, antihypertensive agents, hypoglycemic agents, antiplatelet agents, anticoagulant agents, NIHSS, time from onset of symptoms to admission, total cholesterol, high-density cholesterol lipoprotein, fasting blood glucose, estimated glomerular filtration rate, and high sensitivity C-reactive protein. mRS, modified Rankin Scale.
} 


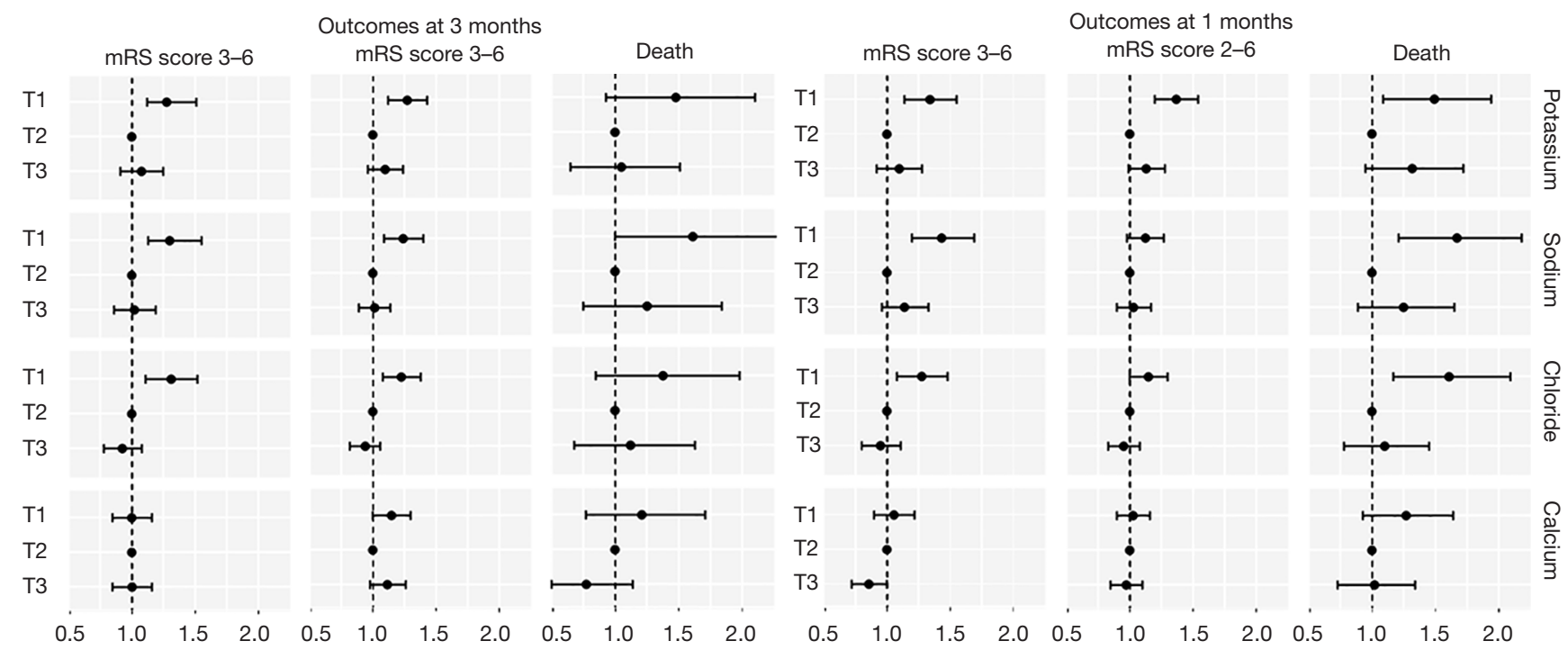

Figure 1 The odds ratio/hazard ratio and 95\% confidence interval of poor functional outcomes (mRS score 3-6/2-6) and all-cause death at 3 months and 1 year by quartiles of potassium, sodium, chloride, and calcium in the adjusted model. Adjusted for age, gender, body mass index, history of hypertension, diabetes mellitus, dyslipidemia, stroke or TIA, atrial fibrillation/flutter, peripheral vascular disease, heart failure, stroke type, TOAST, current smoke, cholesterol-lowering agents, antihypertensive agents, hypoglycemic agents, antiplatelet agents, anticoagulant agents, NIHSS, total cholesterol, high-density cholesterol lipoprotein, fasting blood glucose, estimated glomerular filtration rate, and high sensitivity C-reactive protein. mRS, modified Rankin Scale.

according to the levels of potassium, sodium, chloride (Figure S1 in the online-only data supplement). However, calcium was not related to poor functional outcome (mRS score 3-6) in above analyses (Figure 2D). These relationships were consistent at 3 months (Table 2, Figure 1, and Figure $\mathrm{S} 1$ in the online-only data supplement).

Similar results were observed for the association of hypokalemia (adjusted OR, 1.37; 95\% CI, 1.15-1.63), hyponatremia (adjusted OR, 1.66; 95\% CI, 1.22-2.25), hypochloremia (adjusted OR, 1.59; 95\% CI, 1.24-2.04) and hypocalcemia (adjusted OR, 1.09; 95\% CI, 0.95-1.25) with poor functional outcome (mRS score 3-6) in the secondary analysis (Tables S3-S6 in the online-only data supplement). Additional, hyperkalemia was related to $46 \%$ higher risk of mRS score 3-6 at 1 year (Table S3 in the online-only data supplement).

Same pattern of results was observed when poor functional outcome was defined as mRS score 2-6 except that calcium in the first tertile (adjusted OR, 1.14; $95 \%$ CI, 1.00-1.30) was slightly related to a higher risk of poor functional outcome (mRS score 2-6) at 3 months (Table 2, Figure 2E,F,G,H, Tables S3-S7, and Figure S1 in the onlineonly data supplement).

\section{Associations of electrolytes and all-cause death}

A total of $158(1.53 \%)$ and 325 (3.16) patients ended up with death at 3 months and 1 year follow-up. In the adjusted model, the relationship of the first tertile of potassium, sodium and chloride were also associated with elevated risk of all-cause death at 1 year follow-up, the adjusted HRs with 95\% CIs were 1.45 (1.09-1.94), 1.62 (1.21-2.18) and $1.56(1.17-2.09)$, respectively. No significant association was presented for calcium (Table 3, Figure 1).

Secondary analysis for the relationship of hypoelectrolytemia and all-cause death at 1 year yielded the similar results. Hyponatremia (adjusted HR, 1.58; 95\% CI, 1.16-2.15), hyponatremia (adjusted HR, 2.02; 95\% CI, 1.30-3.15), and hypochloremia (adjusted HR, 1.86; 95\% CI, 1.26-2.77) were associated with increased risk of all-cause (Tables S3-S6 in the online-only data supplement). We also found that hyperkalemia (adjusted HR, 1.87; 95\% CI, 1.143.07) increased the risk of all-cause death. Nevertheless, there was no significant association of hypocalcemia with all-cause mortality (Table 3 and Table S6 in the online-only data supplement). Subgroup analysis stratified by stroke subtypes showed the association between electrolytes and all-cause mortality was consistent across patients with IS 

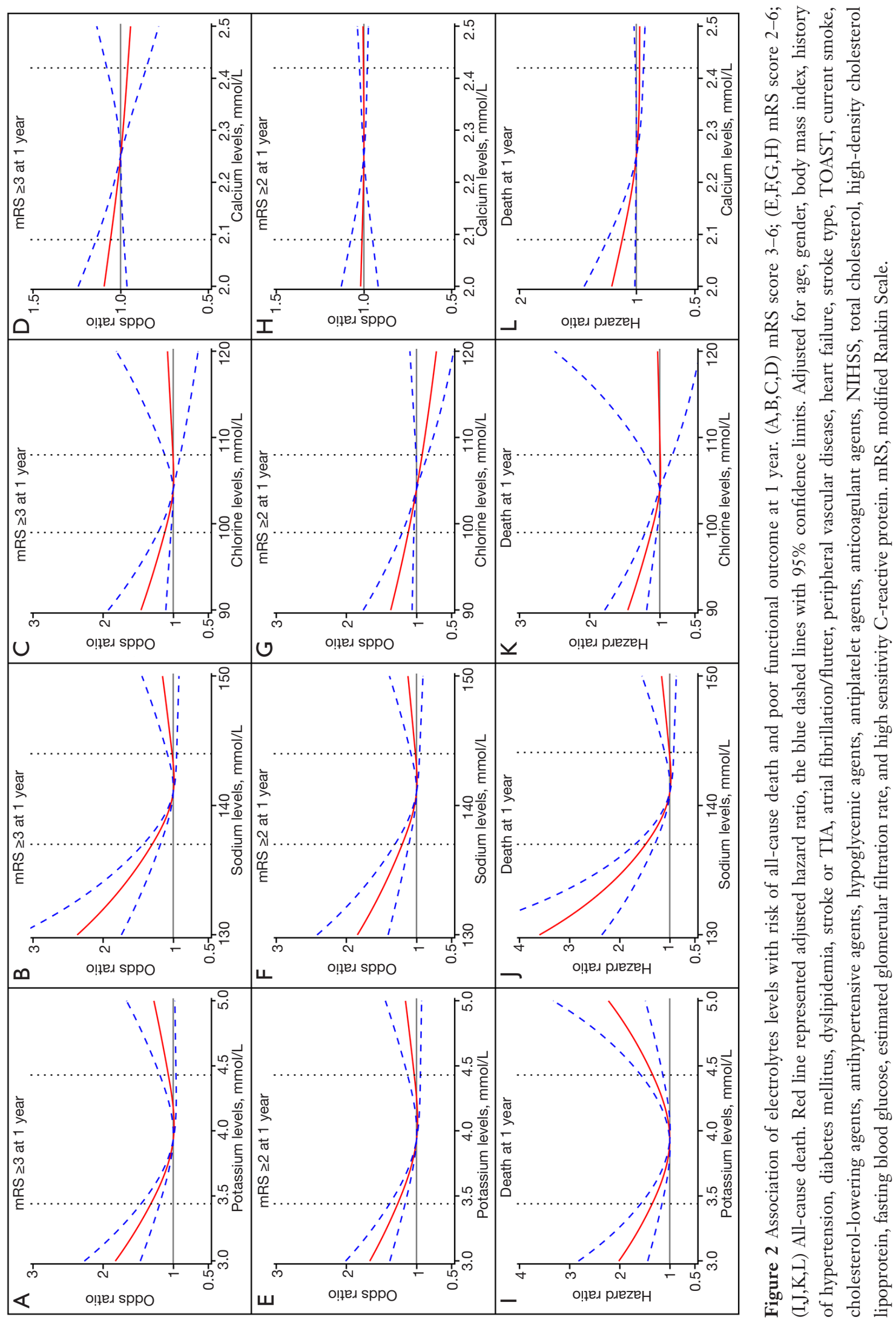
Table 3 Adjusted hazard ratio (95\% confidence interval) for all-cause mortality according to tertiles of serum electrolyte indicators

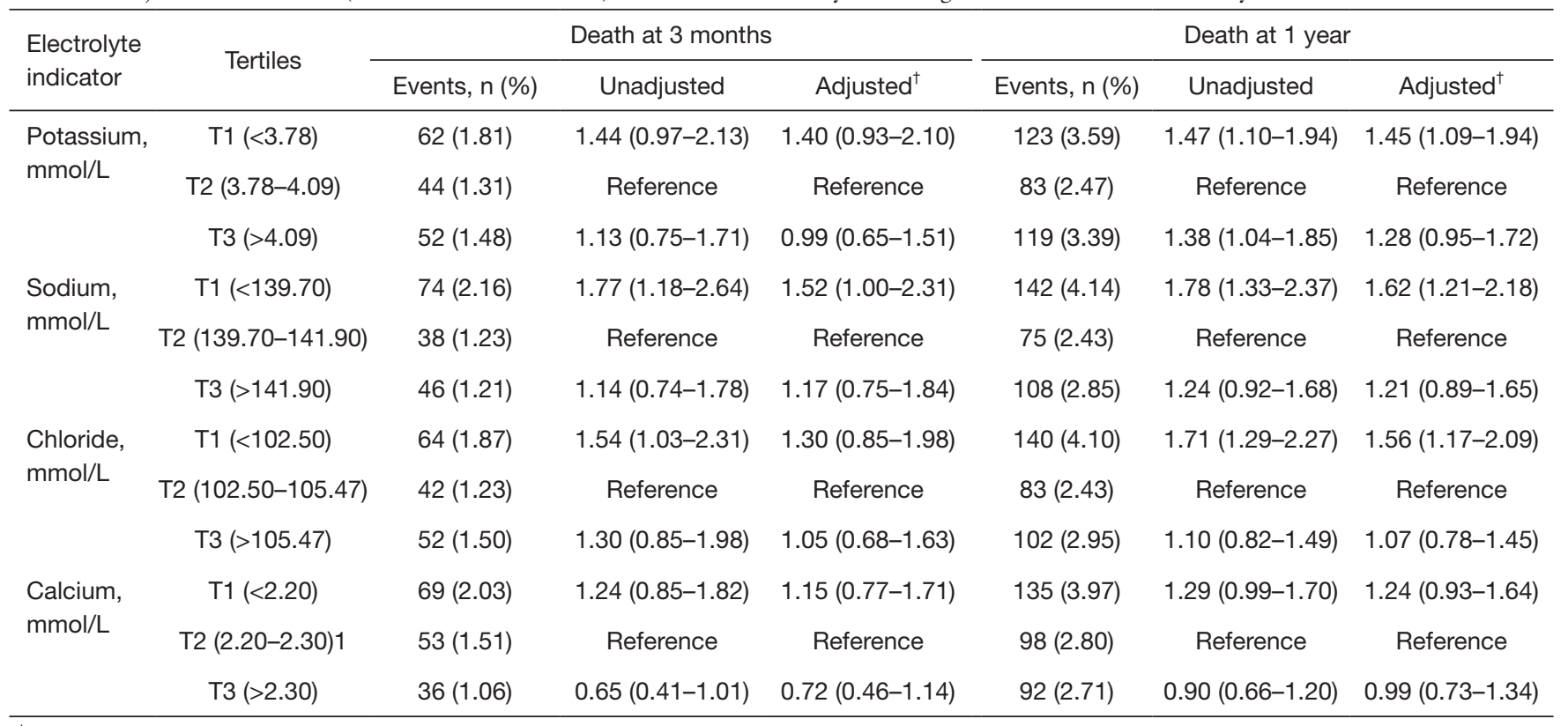

${ }^{\dagger}$, adjusted for age, gender, body mass index, history of hypertension, diabetes mellitus, dyslipidemia, stroke or TIA, atrial fibrillation/ flutter, peripheral vascular disease, heart failure, stroke type, TOAST, current smoke, cholesterol-lowering agents, antihypertensive agents, hypoglycemic agents, antiplatelet agents, anticoagulant agents, NIHSS, time from onset of symptoms to admission, total cholesterol, highdensity cholesterol lipoprotein, fasting blood glucose, estimated glomerular filtration rate, and high sensitivity C-reactive protein.

and TIA (P for interaction $>0.05$ for all).

Consistently, multivariable-adjusted spline regression showed a U-shaped association for potassium, L-shaped associations for sodium and chloride, and no significant association for calcium with all-cause death (Figure 2I, $\mathcal{F}, K, L$ ).

Moreover, all these relationships were consistent at 3 months, except the effect of hypokalemia on all-cause death disappeared (Table 3 and Tables S3-S6 in the onlineonly data supplement).

\section{Discussion}

The major finding of the current study was that lower levels of potassium, sodium and chloride were significantly associated with elevated risk of poor functional outcomes and death in patients with AIS or TIA. However, almost no significant association was present of calcium with these outcomes.

Hypokalemia is a common electrolyte disorder and complication of hospitalized patients, and a lower level of serum potassium has been proved to be correlated with increased ischemic stroke risk (23). Nevertheless, evidence surrounding the relationship between serum potassium and the prognosis of stroke was limited and conflicting. A retrospective study of 361 patients with AIS showed that hypokalemia at the initial admission was associated with a worse poor prognosis with respect to the mRS score 3-6 following first-ever AIS (11). An observational study with 421 stroke patients revealed that a lower plasma potassium at admission was associated with the 3 -month mortality rate of AIS (12). While another observational study of 475 patients demonstrated no association was found between serum potassium levels and short- and longterm outcomes of AIS (13). The discrepancy might be due to the differences in the population characteristics, and the small sample of these studies. Our analyses consisted of 10,299 patients with AIS or TIA had more power and suggested that hypokalemia was a predictor of all-cause death and poor functional outcomes for stroke. Possible mechanism may be that hypokalemia could reduce conductance hyperpolarization in potassium channel of cells, promote formation of free radicals and endothelial dysfunction (24), and the influence of potassium on stroke outcomes could be related directly to the physiological effect of lower extracellular potassium concentration on membrane depolarization leading to events such as a vasoconstriction and deterioration of neurological function 
$(12,24,25)$. Hyperkalemia is a frequent clinical abnormality in patients with chronic kidney disease (26). Epidemiological and clinical data have identified hyperkalemia was a potential risk factor for poor outcomes of acute myocardial infarction and chronic kidney disease $(27,28)$. Results from our study showed that hyperkalemia was associated with allcause death and poor functional outcome regarding to $\mathrm{mRS}$ score 3-6 at 1 year, while the associations disappeared for other outcomes and when timepoint was set at 3 months, which indicated the prognostic role of hyperkalemia may be extent to patients with AIS or TIA in some degree, and it still needed further investigations.

Although hyponatremia has gained wide recognition as a predictor of long-term mortality after stroke, the effect of hyponatremia on short-term mortality was inconsistent (14-17). Rodrigues et al. and a meta-analysis study reported that hyponatremia was related to increased mortality inhospital and at 3 months in IS patients $(14,15)$. Whereas two other studies showed that hyponatremia was not associated with either in-hospital, 30- or 90-day mortality after IS $(16,17)$. The variation among those studies may be explained by many factors, such as inclusion criteria, ethnicity, and sample size. Findings of our study confirmed that hyponatremia was a predictor of all-cause death at 3 months and 1 year. Moreover, we also found hyponatremia was associated with poor functional outcomes of AIS or TIA, which has not been investigated well in previous studies. Mechanisms of the enhanced mortality and poor functional outcomes in hyponatremic patients would be hyponatremia may promote cerebral edema, increase intracranial pressure and then affect cerebral perfusion, which led to vascular injury after stroke $(15,23,29)$.

The association of lower chloride levels and mortality has been shown in chronic kidney disease, hypertensive and postoperative patients $(9,30,31)$. Nevertheless, literatures on prognostic role of hypochloremia in stroke were scarce, to our best knowledge, only one study with 3,314 patients showed hypochloremia at admission was independently associated with in-hospital mortality in AIS patients (16). The significant associations of hypochloremia and all-cause death and poor functional outcomes in our study suggested that hypochloremia could be used as one of the predictors of adverse outcomes of stroke in clinical practice. Possible mechanisms underlying the relationship of hypochloremia and poor prognosis of stroke may be that lower chloride might upregulate inflammatory cytokines and reflect a high anion gap and high rennin level, which may lead to poor outcomes and mortality after stroke indirectly $(32,33)$.
Hypocalcemia is common in critically ill patients, and it has been considered as an indicator of poor prognosis for acute pancreatitis (10). Up to date, the association of calcium with clinical outcomes in patients with AIS or TIA has not been investigated yet. In our study, almost no significant relationships were present for calcium and these outcomes, except the slight association with mRS score 2-6 at 3 months. Possible mechanism for the insignificant associations may be that calcium works in combination with other ions such as potassium, sodium, and magnesium to provide an ionic balance to the vascular membrane and vasodilatation (34).

The strengths of our study include that the study is a large multicenter registry with more than 10,000 patients, supporting sufficient statistical power. Nevertheless, there were still some limitations on our study. Firstly, changes in electrolyte balance have been claimed to play a role in the pathophysiology of ischemic event (35). Clinical data found that a modified serum electrolyte pattern characterized by hypokalemia, hyponatremia, and hypochloremia was more common than hyperkalemia, hypernatremia, and hyperchloremia in stroke patients $(36,37)$. However, our analysis only focused on baseline electrolytes and without accounting for the dynamic changes in electrolytes, which could provide more valuable information to understand the mechanism of the associations. Secondly, equipment heterogeneity at each research centers may lead to biased results, while this may have little impact, since the equipment in each research center is under strict quality control in daily use.

\section{Conclusions}

The study showed that lower levels of potassium, sodium, chloride but not calcium were independent predictors of allcause death and poor functional outcomes in patients with AIS and TIA. The findings of our study may promote their potential use in clinical practice as markers for outcomes in stroke.

\section{Acknowledgments}

We thank all the participating hospitals, their physicians and nurses, and CNSR III steering Committee members and all the participants in the present study.

Funding: This work was supported by grants from National Key R\&D Program of China (2016YFC0901002, 2017YFC1310901, 2018YFC1312903), Beijing Municipal 
Science \& Technology Commission (D171100003017002), National Science and Technology Major Project (2017ZX09304018), Beijing Municipal Administration of Hospitals Incubating Program (PX2020021), Beijing Excellent Talents Training Program (2018000021469G234), and Young Elite Scientists Sponsorship Program by CAST (2018QNRC001).

\section{Footnote}

Reporting Checklist: The authors have completed the STROBE reporting checklist. Available at http://dx.doi. org/10.21037/atm-21-741

Data Sharing Statement: Available at http://dx.doi. org/10.21037/atm-21-741

Conflicts of Interest: All authors have completed the ICMJE uniform disclosure form (available at http://dx.doi. org/10.21037/atm-21-741). The authors have no conflicts of interest to declare.

Ethical Statement: The authors are accountable for all aspects of the work in ensuring that questions related to the accuracy or integrity of any part of the work are appropriately investigated and resolved. The study was conducted in accordance with the Declaration of Helsinki (as revised in 2013). The study was approved by the ethics committee of Beijing Tiantan Hospital (No.: KY2015001-01) and all study centers gave ethical approval of the study protocol. Written consents were obtained from all participants or their legal representatives.

Open Access Statement: This is an Open Access article distributed in accordance with the Creative Commons Attribution-NonCommercial-NoDerivs 4.0 International License (CC BY-NC-ND 4.0), which permits the noncommercial replication and distribution of the article with the strict proviso that no changes or edits are made and the original work is properly cited (including links to both the formal publication through the relevant DOI and the license). See: https://creativecommons.org/licenses/by-nc-nd/4.0/.

\section{References}

1. Wang Y, Liu M, Pu C. 2014 Chinese guidelines for secondary prevention of ischemic stroke and transient ischemic attack. Int J Stroke 2017;12:302-20.
2. Mendis S, Davis S, Norrving B. Organizational update: the world health organization global status report on noncommunicable diseases 2014; one more landmark step in the combat against stroke and vascular disease. Stroke 2015;46:e121-2.

3. Satirapoj B, Kongthaworn S, Choovichian P, et al. Electrolyte disturbances and risk factors of acute kidney injury patients receiving dialysis in exertional heat stroke. BMC Nephrol 2016;17:55.

4. Kim ED, Watt J, Tereshchenko LG, et al. Associations of serum and dialysate electrolytes with QT interval and prolongation in incident hemodialysis: the Predictors of Arrhythmic and Cardiovascular Risk in End-Stage Renal Disease (PACE) study. BMC Nephrol 2019;20:133.

5. Belzile M, Pouliot A, Cumyn A, et al. Renal physiology and fluid and electrolyte disorders in pregnancy. Best Pract Res Clin Obstet Gynaecol 2019;57:1-14.

6. Desai AS, Liu J, Pfeffer MA, et al. Incident Hyperkalemia, Hypokalemia, and Clinical Outcomes During Spironolactone Treatment of Heart Failure With Preserved Ejection Fraction: Analysis of the TOPCAT Trial. J Card Fail 2018;24:313-20.

7. Ma QQ, Fan XD, Li T, et al. Short- and long-term prognostic value of hyponatremia in patients with acute coronary syndrome: A systematic review and meta-analysis. PLoS One 2018;13:e193857.

8. Carcel C, Sato S, Zheng D, et al. Prognostic Significance of Hyponatremia in Acute Intracerebral Hemorrhage: Pooled Analysis of the Intensive Blood Pressure Reduction in Acute Cerebral Hemorrhage Trial Studies. Crit Care Med 2016;44:1388-94.

9. McCallum L, Jeemon P, Hastie CE, et al. Serum chloride is an independent predictor of mortality in hypertensive patients. Hypertension 2013;62:836-43.

10. Kelly A, Levine MA. Hypocalcemia in the critically ill patient. J Intensive Care Med 2013;28:166-77.

11. Gao F, Wang CT, Chen C, et al. Effect of Hypokalemia on Functional Outcome at 3 Months Post-Stroke Among First-Ever Acute Ischemic Stroke Patients. Med Sci Monit 2017;23:2825-32.

12. Gariballa SE, Robinson TG, Fotherby MD. Hypokalemia and potassium excretion in stroke patients. J Am Geriatr Soc 1997;45:1454-58.

13. Fofi L, Dall'armi V, Durastanti L, et al. An observational study on electrolyte disorders in the acute phase of ischemic stroke and their prognostic value. J Clin Neurosci 2012;19:513-6.

14. Rodrigues B, Staff I, Fortunato G, et al. Hyponatremia 
in the prognosis of acute ischemic stroke. J Stroke

Cerebrovasc Dis 2014;23:850-4.

15. Chen Z, Jia Q, Liu C. Association of Hyponatremia and Risk of Short- and Long-Term Mortality in Patients with Stroke: A Systematic Review and Meta-Analysis. J Stroke Cerebrovasc Dis 2019;28:1674-83.

16. Bei HZ, You SJ, Zheng D, et al. Prognostic role of hypochloremia in acute ischemic stroke patients. Acta Neurol Scand 2017;136:672-9.

17. Huang WY, Weng WC, Peng TI, et al. Association of hyponatremia in acute stroke stage with three-year mortality in patients with first-ever ischemic stroke. Cerebrovasc Dis 2012;34:55-62.

18. Wang Y, Jing J, Meng X, et al. The Third China National Stroke Registry (CNSR-III) for patients with acute ischaemic stroke or transient ischaemic attack: design, rationale and baseline patient characteristics. Stroke Vasc Neurol 2019;4:158-64.

19. Adams HP Jr, Bendixen BH, Kappelle LJ, et al. Classification of subtype of acute ischemic stroke. Definitions for use in a multicenter clinical trial. TOAST. Trial of Org 10172 in Acute Stroke Treatment. Stroke 1993;24:35-41.

20. Li W, Chen Z, Ruan W, et al. A meta-analysis of cohort studies including dose-response relationship between shift work and the risk of diabetes mellitus. Eur J Epidemiol 2019;34:1013-24.

21. Pfortmueller CA, Funk GC, Leichtle AB, et al. Electrolyte disorders and in-hospital mortality during prolonged heat periods: a cross-sectional analysis. PLoS One 2014;9:e92150.

22. Goad NT, Bakhru RN, Pirkle JL, et al. Association of Hyperchloremia With Unfavorable Clinical Outcomes in Adults With Diabetic Ketoacidosis. J Intensive Care Med 2020;35:1307-13.

23. Mattsson N, Nielsen OW, Johnson L, et al. Prognostic Impact of Mild Hypokalemia in Terms of Death and Stroke in the General Population-A Prospective Population Study. Am J Med 2018;131:318.e9-19.

24. Cheng CJ, Kuo E, Huang CL. Extracellular potassium homeostasis: insights from hypokalemic periodic paralysis. Semin Nephrol 2013;33:237-47.

Cite this article as: Wang A, Tian X, Gu H, Zuo Y, Meng X, Chen P, Li H, Wang Y. Electrolytes and clinical outcomes in patients with acute ischemic stroke or transient ischemic attack. Ann Transl Med 2021;9(13):1069. doi: 10.21037/atm-21-741
25. Rodan AR. Potassium: friend or foe? Pediatr Nephrol 2017;32:1109-21.

26. Kovesdy CP. Updates in hyperkalemia: Outcomes and therapeutic strategies. Rev Endocr Metab Disord 2017;18:41-7.

27. Grodzinsky A, Goyal A, Gosch K, et al. Prevalence and Prognosis of Hyperkalemia in Patients with Acute Myocardial Infarction. Am J Med 2016;129:858-65.

28. Nakhoul GN, Huang H, Arrigain S, et al. Serum Potassium, End-Stage Renal Disease and Mortality in Chronic Kidney Disease. Am J Nephrol 2015;41:456-63.

29. Ayus JC, Achinger SG, Arieff A. Brain cell volume regulation in hyponatremia: role of sex, age, vasopressin, and hypoxia. Am J Physiol Renal Physiol 2008;295:F61924.

30. Mandai S, Kanda E, Iimori S, et al. Association of serum chloride level with mortality and cardiovascular events in chronic kidney disease: the CKD-ROUTE study. Clin Exp Nephrol 2017;21:104-11.

31. Kimura S, Matsumoto S, Muto N, et al. Association of serum chloride concentration with outcomes in postoperative critically ill patients: a retrospective observational study. J Intensive Care 2014;2:39.

32. Yang H, Huang LY, Zeng DY, et al. Decrease of intracellular chloride concentration promotes endothelial cell inflammation by activating nuclear factor- B pathway. Hypertension 2012;60:1287-93.

33. Taylor EN, Forman JP, Farwell WR. Serum anion gap and blood pressure in the national health and nutrition examination survey. Hypertension 2007;50:320-4.

34. Houston MC, Harper KJ. Potassium, magnesium, and calcium: their role in both the cause and treatment of hypertension. J Clin Hypertens (Greenwich) 2008;10:3-11.

35. Martha SR, Fraser J, Pennypacker K. Acid-Base and Electrolyte Changes Drive Early Pathology in Ischemic Stroke. Neuromolecular Med 2019;21:540-5.

36. Alam MN, Uddin M, Rahman K, et al. Electrolyte changes in stroke. Mymensingh Med J 2012;21:594-9.

37. Solini A, Zamboni P, Passaro A, et al. Acute vascular events and electrolytes variations in elderly patients. Horm Metab Res 2006;38:197-202. 
Table S1 Comparison of baseline characteristics of participants and nonparticipants

\begin{tabular}{|c|c|c|c|}
\hline Characteristics & Nonparticipants, $\mathrm{N}=4867$ & Participants, $N=10299$ & $P$ value \\
\hline Age, y & $62(54-70)$ & $63(54-70)$ & 0.5865 \\
\hline Men, n (\%) & $3384(69.53)$ & $6980(67.77)$ & 0.0300 \\
\hline $\mathrm{BMI}, \mathrm{kg} / \mathrm{m}^{2}$ & 24.47 (22.72-26.49) & $24.49(22.58-26.57)$ & 0.8171 \\
\hline \multicolumn{4}{|l|}{ Medical History, n (\%) } \\
\hline Hypertension & 3056 (62.79) & $6438(62.51)$ & 0.7400 \\
\hline Diabetes mellitus & $1145(23.53)$ & 2365 (22.96) & 0.4433 \\
\hline Dyslipidemia & $322(6.62)$ & $869(8.44)$ & 0.0001 \\
\hline Stroke or TIA & $1111(22.83)$ & $2244(21.79)$ & 0.1502 \\
\hline Atrial fibrillation/flutter & $305(6.27)$ & $714(6.93)$ & 0.1262 \\
\hline Peripheral vascular disease & $44(0.90)$ & $74(0.72)$ & 0.2248 \\
\hline Heart failure & $25(3.94)$ & $69(4.62)$ & 0.4863 \\
\hline \multicolumn{4}{|l|}{ Stroke type/Subtype, n (\%) } \\
\hline Ischemic stroke & 4571 (93.92) & $9575(92.97)$ & 0.0296 \\
\hline TIA & $296(6.08)$ & $724(7.03)$ & \\
\hline \multicolumn{4}{|l|}{ TOAST, n (\%) } \\
\hline Large-artery atherosclerosis & $1256(25.81)$ & $2600(25.25)$ & 0.018 \\
\hline Cardioembolism & $284(5.84)$ & $633(6.15)$ & \\
\hline Small-vessel occlusion & $1079(22.17)$ & $2086(20.25)$ & \\
\hline Other determined etiology & $48(0.99)$ & $134(1.30)$ & \\
\hline Undetermined etiology & $2200(45.20)$ & $4846(47.05)$ & \\
\hline Current smoker, n (\%) & $1552(31.89)$ & $3200(31.07)$ & 0.4719 \\
\hline \multicolumn{4}{|l|}{ Medication in hospital, $\mathrm{n}(\%)$} \\
\hline Cholesterol-lowering agents & $4693(97.20)$ & $9813(95.97)$ & 0.0002 \\
\hline Antihypertensive agents & $2216(45.90)$ & $4784(46.79)$ & 0.3077 \\
\hline Hypoglycemic agents & $1237(25.62)$ & 2555 (24.99) & 0.4032 \\
\hline Antiplatelet agents & $4678(96.89)$ & 9935 (97.16) & 0.3574 \\
\hline Anticoagulant agents & $485(10.05)$ & $1061(10.38)$ & 0.5324 \\
\hline NIHSS score on admission & $3(1-6)$ & $3(1-6)$ & 0.0701 \\
\hline \multicolumn{4}{|l|}{ Lipid level } \\
\hline $\mathrm{TC}, \mathrm{mmol} / \mathrm{L}$ & $3.89(3.26-4.63)$ & $4.02(3.35-4.78)$ & $<0.0001$ \\
\hline LDL, mmol/L & $2.26(1.71-2.92)$ & $2.34(1.74-3.01)$ & 0.0003 \\
\hline $\mathrm{HDL}, \mathrm{mmol} / \mathrm{L}$ & $0.92(0.76-1.10)$ & $0.94(0.78-1.13)$ & $<0.0001$ \\
\hline $\mathrm{TG}, \mathrm{mmol} / \mathrm{L}$ & $1.36(1.03-1.87)$ & $1.37(1.03-1.87)$ & 0.8430 \\
\hline $\mathrm{FBG}, \mathrm{mmol} / \mathrm{L}$ & $5.48(4.84-6.80)$ & $5.56(4.90-6.91)$ & 0.0001 \\
\hline eGFR, mL/min/1.73 m² & 93.47 (82.56-101.96) & $92.93(80.85-101.84)$ & 0.0378 \\
\hline Hs-CRP, mg/L & $1.67(0.81-4.51)$ & $1.84(0.82-4.83)$ & 0.0126 \\
\hline Potassium, mmol/L & $3.90(3.68-4.15)$ & $3.93(3.68-4.19)$ & 0.0561 \\
\hline Sodium, mmol/L & $140.40(138.90-1.42 .00)$ & $141.00(139.00-142.70)$ & 0.0003 \\
\hline Chloride, $\mathrm{mmol} / \mathrm{L}$ & $105.00(102.30-107.00)$ & $104.00(101.70-106.10)$ & $<0.0001$ \\
\hline Calcium, mmol/L & $2.23(2.16-2.32)$ & $2.25(2.17-2.34)$ & 0.0625 \\
\hline
\end{tabular}

Continuous variables are expressed as median with interquartile range. Categorical variables are expressed as frequency with percentage. BMI, body mass index; TIA, transient ischemic Attack; TOAST, Trial of Org 10172 in Acute Stroke Treatment; NIHSS, The National Institutes of Health Stroke Scale; TC, total cholesterol; LDL, low-density lipoprotein cholesterol; HDL, high-density lipoprotein cholesterol; TG, triglycerides; FBG, fasting blood glucose; eGFR, estimated glomerular filtration rate; hs-CRP, high sensitivity C-reactive protein. 
Table S2 Baseline characteristics of patients according to functional outcome at 1 year

\begin{tabular}{|c|c|c|c|c|c|c|}
\hline Characteristics & mRS score $<3(n=8903)$ & mRS score $\geq 3(n=1396)$ & $P$ value & mRS score $<2(n=7796)$ & mRS score $\geq 2(n=2503)$ & $P$ value \\
\hline Age, y & $62(54-69)$ & $68(60-76)$ & $<0.0001$ & $62(53-68)$ & $66(59-75)$ & $<0.0001$ \\
\hline Men, n (\%) & $6136(68.92)$ & $844(60.46)$ & $<0.0001$ & 5410 (69.39) & $1570(62.72)$ & $<0.0001$ \\
\hline BMI, $\mathrm{kg} / \mathrm{m}^{2}$ & $24.51(22.67-26.57)$ & $24.22(22.03-26.42)$ & $<0.0001$ & $24.52(22.76-26.64)$ & $24.22(22.09-26.35)$ & $<0.0001$ \\
\hline \multicolumn{7}{|l|}{ Medical History, n (\%) } \\
\hline Hypertension & $5501(61.79)$ & $937(67.12)$ & 0.0001 & $4765(61.12)$ & $1673(66.84)$ & $<0.0001$ \\
\hline Diabetes mellitus & $1997(22.43)$ & $368(26.36)$ & 0.0012 & $1681(21.56)$ & $684(27.33)$ & $<0.0001$ \\
\hline Dyslipidemia & $736(8.27)$ & $133(9.53)$ & 0.1152 & $662(8.49)$ & $207(8.27)$ & 0.7287 \\
\hline Stroke or TIA & $1778(19.97)$ & $466(33.38)$ & $<0.0001$ & 1475 (18.92) & 769 (30.72) & $<0.0001$ \\
\hline Atrial fibrillation/flutter & $506(5.68)$ & $208(14.90)$ & $<0.0001$ & $411(5.27)$ & $303(12.11)$ & $<0.0001$ \\
\hline Peripheral vascular disease & $57(0.64)$ & $17(1.22)$ & 0.0175 & $47(0.60)$ & $27(1.08)$ & 0.0142 \\
\hline Heart failure & $51(4.32)$ & $18(5.75)$ & 0.2845 & $32(3.24)$ & $37(7.31)$ & 0.0004 \\
\hline \multicolumn{7}{|l|}{ Stroke type/Subtype, n (\%) } \\
\hline Ischemic stroke & $8203(92.14)$ & $1372(98.28)$ & $<0.0001$ & $7127(91.42)$ & $2448(97.80)$ & $<0.0001$ \\
\hline TIA & $700(7.86)$ & $24(1.72)$ & & $669(8.58)$ & $55(2.20)$ & \\
\hline \multicolumn{7}{|l|}{ TOAST, n (\%) } \\
\hline Large-artery atherosclerosis & $2099(23.58)$ & $501(35.89)$ & $<0.0001$ & $1766(22.65)$ & 834 (33.32) & $<0.0001$ \\
\hline Cardioembolism & $510(5.73)$ & $123(8.81)$ & & $434(5.57)$ & $199(7.95)$ & \\
\hline Small-vessel occlusion & $1945(21.85)$ & $141(10.10)$ & & $1772(22.73)$ & $314(12.54)$ & \\
\hline Other determined etiology & $109(1.22)$ & $25(1.79)$ & & $93(1.19)$ & $41(1.64)$ & \\
\hline Undetermined etiology & $4240(47.62)$ & $606(43.41)$ & & $3731(47.86)$ & $1115(44.55)$ & \\
\hline Current smoker, n (\%) & $2883(32.38)$ & $317(22.71)$ & $<0.0001$ & $2559(32.82)$ & $641(25.61)$ & $<0.0001$ \\
\hline \multicolumn{7}{|l|}{ Medication in hospital, n (\%) } \\
\hline Cholesterol-lowering agents & $8483(95.95)$ & $1330(96.10)$ & 0.7952 & 7414 (95.84) & $2399(96.38)$ & 0.2279 \\
\hline Antihypertensive agents & $4085(46.21)$ & $699(50.51)$ & 0.0029 & $3543(45.80)$ & $1241(49.86)$ & 0.0004 \\
\hline Hypoglycemic agents & $2175(24.60)$ & $380(27.46)$ & 0.0225 & $1844(23.84)$ & $711(28.57)$ & $<0.0001$ \\
\hline Antiplatelet agents & $8626(97.57)$ & $1309(94.58)$ & $<0.0001$ & $7554(97.65)$ & $2381(95.66)$ & $<0.0001$ \\
\hline Anticoagulant agents & $847(9.58)$ & $214(15.46)$ & $<0.0001$ & $700(9.05)$ & $361(14.50)$ & $<0.0001$ \\
\hline NIHSS score on admission & $3(1-5)$ & $7(4-11)$ & $<0.0001$ & $3(1-5)$ & $6(3-10)$ & $<0.0001$ \\
\hline \multicolumn{7}{|l|}{ Lipid level } \\
\hline $\mathrm{TC}, \mathrm{mmol} / \mathrm{L}$ & $4.02(3.35-4.78)$ & $3.97(3.31-4.8)$ & 0.5598 & $4.03(3.35-4.77)$ & $3.97(3.33-4.8)$ & 0.5047 \\
\hline $\mathrm{LDL}, \mathrm{mmol} / \mathrm{L}$ & $2.33(1.73-3)$ & $2.37(1.78-3.1)$ & 0.0885 & 2.33 (1.73-2.99) & $2.36(1.76-3.08)$ & 0.0759 \\
\hline $\mathrm{HDL}, \mathrm{mmol} / \mathrm{L}$ & $0.94(0.78-1.13)$ & $0.97(0.79-1.15)$ & 0.0514 & $0.94(0.78-1.13)$ & $0.94(0.78-1.13)$ & 0.6636 \\
\hline $\mathrm{TG}, \mathrm{mmol} / \mathrm{L}$ & $1.39(1.04-1.9)$ & $1.25(0.96-1.69)$ & $<0.0001$ & $1.39(1.04-1.91)$ & $1.3(1-1.76)$ & $<0.0001$ \\
\hline $\mathrm{FBG}, \mathrm{mmol} / \mathrm{L}$ & $5.51(4.9-6.83)$ & $5.81(5.05-7.37)$ & $<0.0001$ & $5.5(4.88-6.77)$ & $5.78(5.02-7.36)$ & $<0.0001$ \\
\hline eGFR, $\mathrm{mL} / \mathrm{min} / 1.73 \mathrm{~m}^{2}$ & $93.61(82.46-102.43)$ & $87.9(71.7-97.68)$ & $<0.0001$ & $94.06(82.72-102.55)$ & 89.27 (74.82-98.88) & $<0.0001$ \\
\hline hs-CRP, mg/L & $1.65(0.79-4.17)$ & $3.93(1.33-12.39)$ & $<0.0001$ & $1.56(0.77-3.91)$ & $3.15(1.17-8.53)$ & $<0.0001$ \\
\hline Potassium, $\mathrm{mmol} / \mathrm{L}$ & $3.94(3.70-4.19)$ & $3.87(3.60-4.17)$ & $<0.0001$ & $3.94(3.70-4.20)$ & $3.89(3.61-4.17)$ & $<0.0001$ \\
\hline Sodium, mmol/L & $141.00(139.00-142.80)$ & $140.20(138.20-142.50)$ & $<0.0001$ & $141.00(139.00-142.80)$ & $140.60(138.50-1.42 .50)$ & $<0.0001$ \\
\hline Chloride, $\mathrm{mmol} / \mathrm{L}$ & $104.0(101.9-106.1)$ & $103.8(101.0-1.06 .0)$ & 0.0003 & $104.00(101.90-106.10)$ & $103.90(101.00-106.10)$ & $<0.0001$ \\
\hline Calcium, mmol/L & $2.25(2.17-2.34)$ & $2.23(2.14-2.32)$ & $<0.0001$ & $2.25(2.17-2.34)$ & $2.24(2.16-2.33)$ & 0.0001 \\
\hline
\end{tabular}

Continuous variables are expressed as median with interquartile range. Categorical variables are expressed as frequency with percentage. mRS, modified Rankin Scale; BMI, body mass index; TIA, transient ischemic Attack; TOAST, Trial of Org 10172 in Acute Stroke Treatment; NIHSS, The National Institutes of Health Stroke Scale; TC, total cholesterol; LDL, low-density lipoprotein cholesterol; HDL, high-density lipoprotein cholesterol; TG, triglycerides; FBG, fasting blood glucose; eGFR, estimated glomerular filtration rate; hs-CRP, high sensitivity C-reactive protein. 


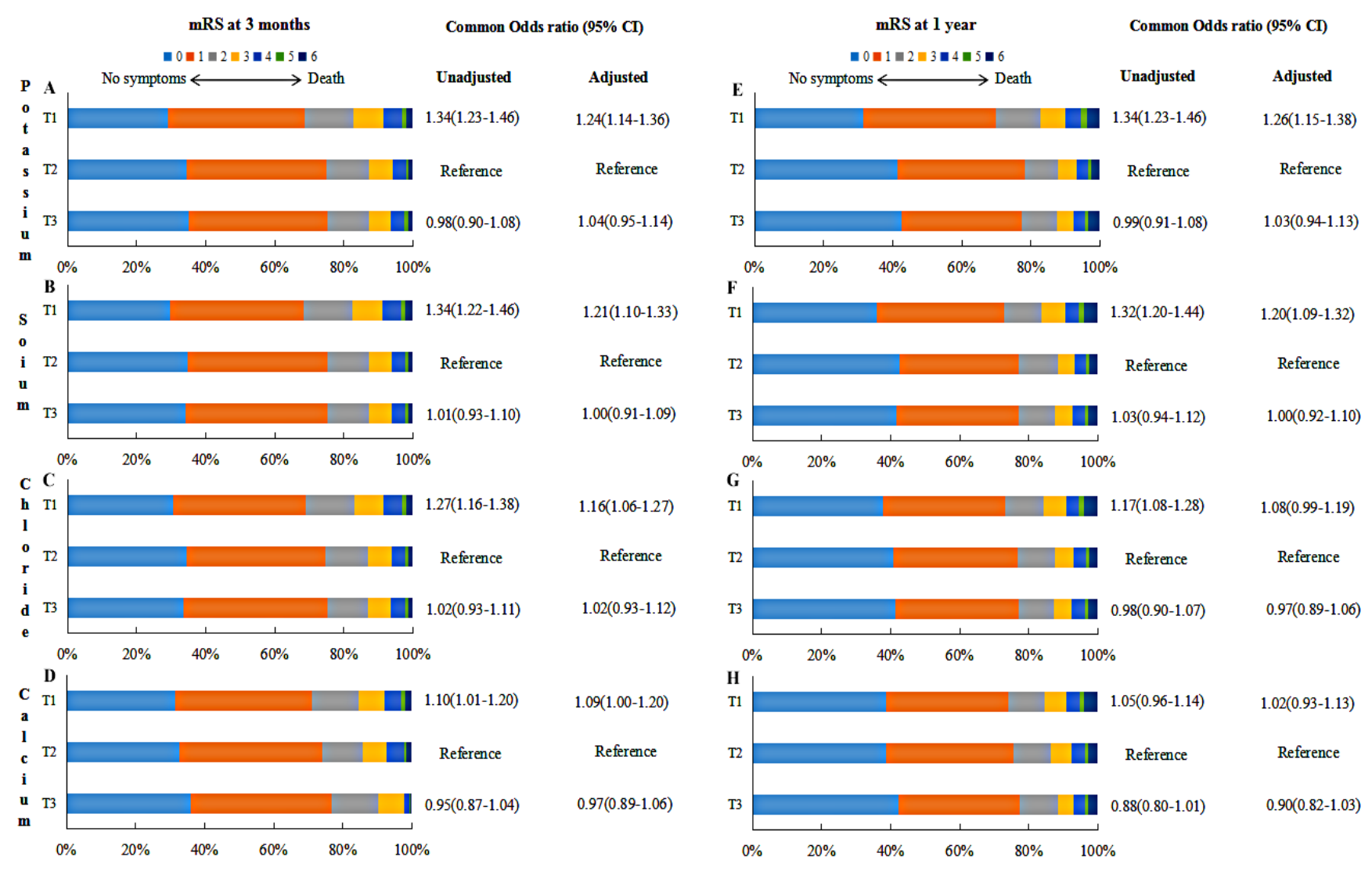

Figure S1 Modified Rankin Scale scores stratified by electrolyte levels. The figure is shown the distribution of scores on the modified Rankin scale. Scores range from 0 to 6, with 0 indicating no symptoms, 1 no clinically significant disability, 2 slight disability, 3 moderate disability, 4 moderately severe disability, 5 severe disability and 6 death. In the multivariate regression model, we adjusted for age, gender, body mass index, history of hypertension, diabetes mellitus, dyslipidemia, stroke or TIA, atrial fibrillation/flutter, peripheral vascular disease, heart failure, stroke type, TOAST, current smoke, cholesterol-lowering agents, antihypertensive agents, hypoglycemic agents, antiplatelet agents, anticoagulant agents, NIHSS, total cholesterol, high-density cholesterol lipoprotein, fasting blood glucose, estimated glomerular filtration rate, and high sensitivity C-reactive protein. mRS, modified Rankin scale; CI, confidence interval. 
Table S3 Association of all-cause death and poor functional outcomes with serum potassium

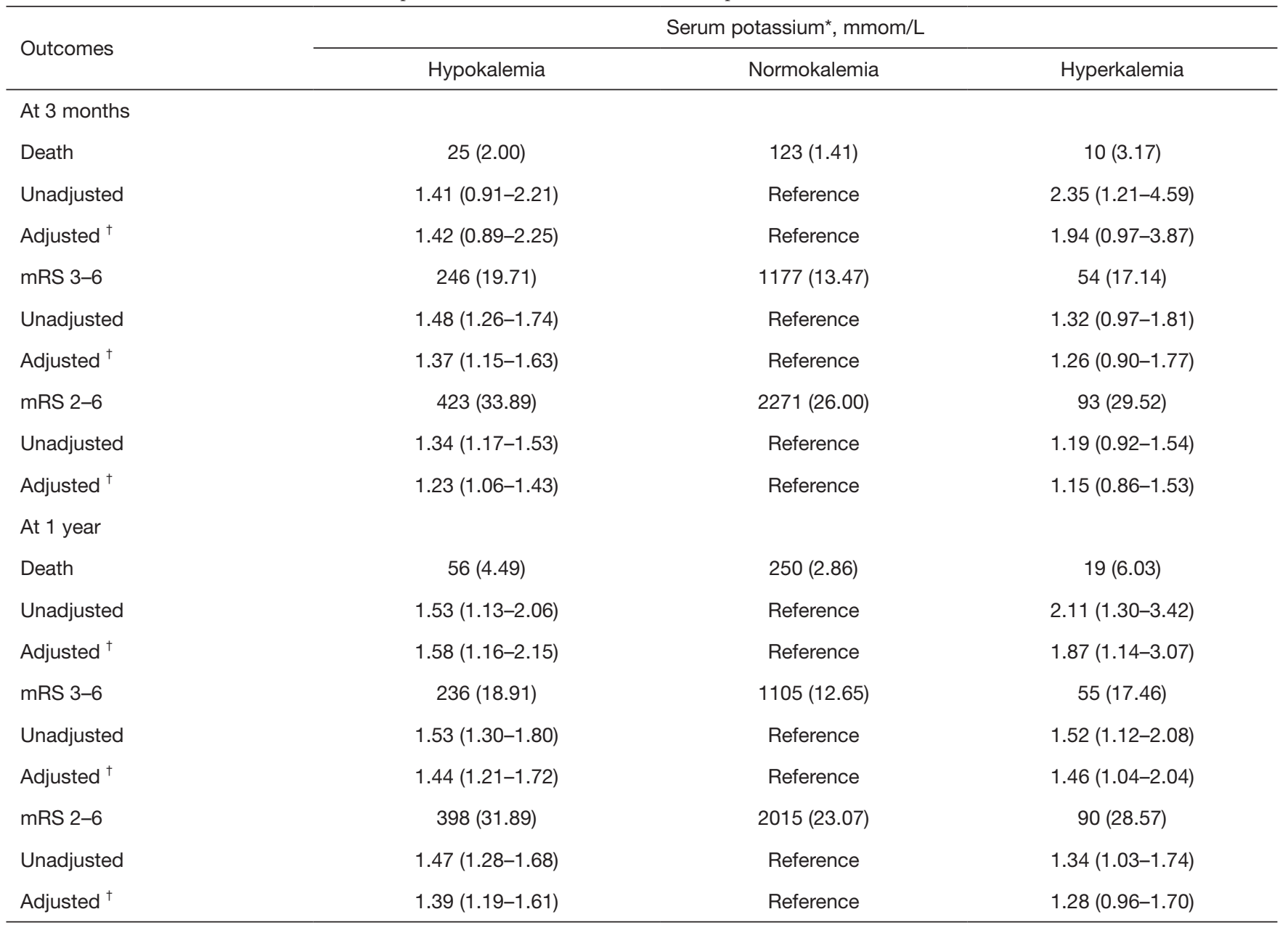

${ }^{*}$ Hypokalemia: potassium less than $3.5 \mathrm{mmol} / \mathrm{L}$; Normokalemia: potassium from 3.5 to $4.7 \mathrm{mmol} / \mathrm{L}$; Hyperkalemia: potassium from more than $4.7 \mathrm{mmol} / \mathrm{L}$. ${ }^{\dagger}$ Adjusted for age, gender, body mass index, history of hypertension, diabetes mellitus, dyslipidemia, stroke or TIA, atrial fibrillation/flutter, peripheral vascular disease, heart failure, stroke type, TOAST, current smoke, cholesterol-lowering agents, antihypertensive agents, hypoglycemic agents, antiplatelet agents, anticoagulant agents, NIHSS, total cholesterol, high-density cholesterol lipoprotein, fasting blood glucose, estimated glomerular filtration rate, and high sensitivity C-reactive protein. mRS, modified Rankin Scale. 
Table S4 Association of all-cause death and poor functional outcomes with serum sodium

\begin{tabular}{|c|c|c|c|}
\hline Outcomes & \multicolumn{3}{|c|}{ Serum sodium, mmom/L } \\
\hline \multicolumn{4}{|l|}{ At 3 months } \\
\hline Death & $17(5.21)$ & $130(1.38)$ & $11(2.11)$ \\
\hline Unadjusted & $3.58(2.06-6.23)$ & Reference & $1.90(0.98-3.69)$ \\
\hline mRS 3-6 & $82(25.15)$ & $1321(13.98)$ & $74(14.20)$ \\
\hline Unadjusted & 2.03 (1.54-2.68) & Reference & $1.02(0.78-1.34)$ \\
\hline Adjusted $^{\dagger}$ & $1.66(1.22-2.25)$ & Reference & $1.03(0.77-1.37)$ \\
\hline mRS 2-6 & $135(41.41)$ & $2510(26.56)$ & $142(27.26)$ \\
\hline \multicolumn{4}{|l|}{ At 1 year } \\
\hline Death & $29(8.90)$ & 279 (2.95) & $17(3.26)$ \\
\hline Unadjusted & $2.98(1.97-4.51)$ & Reference & $1.11(0.66-1.87)$ \\
\hline Adjusted $^{\dagger}$ & $2.02(1.30-3.15)$ & Reference & $0.98(0.58-1.67)$ \\
\hline mRS 3-6 & $76(23.31)$ & $1252(13.25)$ & $68(13.05)$ \\
\hline Unadjusted & $1.91(1.44-2.54)$ & Reference & $0.95(0.71-1.25)$ \\
\hline Adjusted $^{\dagger}$ & $1.53(1.12-2.09)$ & Reference & $0.90(0.67-1.21)$ \\
\hline mRS 2-6 & $117(35.89)$ & 2259 (23.90) & $127(24.38)$ \\
\hline
\end{tabular}

*Hyponatremia: sodium less than $135 \mathrm{mmol} / \mathrm{L}$; Normonatremia: sodium from 135 to $145 \mathrm{mmol} / \mathrm{L}$; Hypernatremia: sodium from more than $145 \mathrm{mmol} / \mathrm{L}$. ${ }^{\dagger}$ Adjusted for age, gender, body mass index, history of hypertension, diabetes mellitus, dyslipidemia, stroke or TIA, atrial fibrillation/flutter, peripheral vascular disease, heart failure, stroke type, TOAST, current smoke, cholesterol-lowering agents, antihypertensive agents, hypoglycemic agents, antiplatelet agents, anticoagulant agents, NIHSS, total cholesterol, high-density cholesterol lipoprotein, fasting blood glucose, estimated glomerular filtration rate, and high sensitivity C-reactive protein. mRS, modified Rankin Scale. 
Table S5 Association of all-cause death and poor functional outcomes with serum chloride

\begin{tabular}{|c|c|c|c|}
\hline Outcomes & \multicolumn{3}{|c|}{ Serum chloride ${ }^{\star}, \mathrm{mmom} / \mathrm{L}$} \\
\hline \multicolumn{4}{|l|}{ At 3 months } \\
\hline Death & $21(3.98)$ & $122(1.32)$ & $15(2.76)$ \\
\hline Unadjusted & 3.03 (1.84-4.98) & Reference & $1.66(0.94-2.94)$ \\
\hline mRS 3-6 & $115(21.78)$ & $1288(13.96)$ & 74 (13.60) \\
\hline Unadjusted & $1.76(1.40-2.22)$ & Reference & $0.84(0.64-1.10)$ \\
\hline Adjusted $^{\dagger}$ & $1.59(1.24-2.04)$ & Reference & $0.80(0.60-1.06)$ \\
\hline mRS 2-6 & $189(35.80)$ & 2459 (26.65) & $139(25.55)$ \\
\hline \multicolumn{4}{|l|}{ At 1 year } \\
\hline Death & $35(6.63)$ & $264(2.86)$ & $26(4.78)$ \\
\hline Unadjusted & $2.25(1.55-3.25)$ & Reference & $1.51(0.98-2.32)$ \\
\hline Adjusted $^{\dagger}$ & $1.86(1.26-2.77)$ & Reference & $1.36(0.87-2.13)$ \\
\hline mRS 3-6 & $110(20.83)$ & 1208 (13.09) & 78 (14.34) \\
\hline Unadjusted & $1.74(1.38-2.19)$ & Reference & $1.00(0.77-1.30)$ \\
\hline Adjusted $^{\dagger}$ & $1.58(1.23-2.04)$ & Reference & $0.92(0.69-1.22)$ \\
\hline mRS 2-6 & $166(31.44)$ & 2205 (23.90) & $132(24.26)$ \\
\hline
\end{tabular}

${ }^{*}$ Hypochloremia: chloride less than $98 \mathrm{mmol} / \mathrm{L}$; Normochloremia: chloride from 98 to $109 \mathrm{mmol} / \mathrm{L}$; Hyperchloremia: chloride from more than $109 \mathrm{mmol} / \mathrm{L}$. ${ }^{\dagger}$ Adjusted for age, gender, body mass index, history of hypertension, diabetes mellitus, dyslipidemia, stroke or TIA, atrial fibrillation/flutter, peripheral vascular disease, heart failure, stroke type, TOAST, current smoke, cholesterol-lowering agents, antihypertensive agents, hypoglycemic agents, antiplatelet agents, anticoagulant agents, NIHSS, total cholesterol, high-density cholesterol lipoprotein, fasting blood glucose, estimated glomerular filtration rate, and high sensitivity C-reactive protein. mRS, modified Rankin Scale. 
Table S6 Association of all-cause death and poor functional outcomes with serum calcium

\begin{tabular}{|c|c|c|c|}
\hline Outcomes & \multicolumn{3}{|c|}{ Serum calcium* ${ }^{\star}$ mmom/L } \\
\hline \multicolumn{4}{|l|}{ At 3 months } \\
\hline Death & $88(1.76)$ & $70(1.36)$ & $0(0.00)$ \\
\hline Unadjusted & $1.22(0.86-1.72)$ & Reference & - \\
\hline mRS 3-6 & $746(14.92)$ & $713(13.83)$ & $18(12.77)$ \\
\hline Unadjusted & $1.03(0.91-1.17)$ & Reference & $1.05(0.62-1.77)$ \\
\hline Adjusted $^{\dagger}$ & $0.96(0.84-1.09)$ & Reference & $0.99(0.57-1.71)$ \\
\hline mRS 2-6 & $1412(28.23)$ & $1339(25.96)$ & $36(25.53)$ \\
\hline \multicolumn{4}{|l|}{ At 1 year } \\
\hline Death & $174(3.48)$ & $145(2.81)$ & $6(4.26)$ \\
\hline Unadjusted & $1.17(0.92-1.49)$ & Reference & $1.43(0.62-3.31)$ \\
\hline Adjusted $^{\dagger}$ & $1.05(0.82-1.35)$ & Reference & $1.64(0.70-3.81)$ \\
\hline mRS 3-6 & 739 (14.78) & $642(12.45)$ & $15(10.64)$ \\
\hline Unadjusted & $1.18(1.04-1.34)$ & Reference & $0.87(0.50-1.52)$ \\
\hline Adjusted $^{\dagger}$ & $1.09(0.95-1.25)$ & Reference & $0.84(0.47-1.50)$ \\
\hline mRS 2-6 & $1276(25.51)$ & $1199(23.25)$ & $28(19.86)$ \\
\hline
\end{tabular}

${ }^{*}$ Hypocalcemia: calcium less than $2.25 \mathrm{mmol} / \mathrm{L}$; Normocalcemia: calcium from 2.25 to $2.58 \mathrm{mmol} / \mathrm{L}$; Hypercalcemia: calcium from more than $2.58 \mathrm{mmol} / \mathrm{L} .{ }^{\dagger}$ Adjusted for age, gender, body mass index, history of hypertension, diabetes mellitus, dyslipidemia, stroke or TIA, atrial fibrillation/flutter, peripheral vascular disease, heart failure, stroke type, TOAST, current smoke, cholesterol-lowering agents, antihypertensive agents, hypoglycemic agents, antiplatelet agents, anticoagulant agents, NIHSS, total cholesterol, high-density cholesterol lipoprotein, fasting blood glucose, estimated glomerular filtration rate, and high sensitivity C-reactive protein. mRS, modified Rankin Scale. 
Table S7 Adjusted odds ratio (95\% confidence interval) for poor functional outcome (mRS score 2-6) according to tertiles of serum electrolyte indicators

\begin{tabular}{|c|c|c|c|c|c|c|c|}
\hline $\begin{array}{l}\text { Electrolyte } \\
\text { indicator }\end{array}$ & Tertiles & \multicolumn{3}{|c|}{ mRS score $2-6$ at 3 months } & \multicolumn{3}{|c|}{$\mathrm{mRS}$ score $2-6$ at 1 year } \\
\hline \multirow[t]{3}{*}{ Potassium } & $\mathrm{T} 1$ & 1075 (31.38) & $1.32(1.18-1.48)$ & $1.27(1.12-1.43)$ & $976(28.49)$ & $1.40(1.25-1.57)$ & $1.36(1.20-1.54)$ \\
\hline & $\mathrm{T} 2$ & $838(24.93)$ & Reference & Reference & $733(21.80)$ & Reference & Reference \\
\hline & T3 & $874(24.89)$ & $1.06(0.95-1.19)$ & $1.09(0.96-1.24)$ & $794(22.61)$ & $1.10(0.98-1.24)$ & $1.12(0.99-1.28)$ \\
\hline \multirow{2}{*}{ Sodium } & $\mathrm{T} 2$ & $762(24.70)$ & Reference & Reference & $710(23.01)$ & Reference & Reference \\
\hline & T3 & $943(24.89)$ & $0.99(0.88-1.12)$ & $1.01(0.89-1.14)$ & $864(22.81)$ & $1.01(0.90-1.14)$ & $1.02(0.90-1.17)$ \\
\hline \multirow[t]{2}{*}{ Chloride } & $\mathrm{T} 1$ & $1063(31.10)$ & $1.31(1.17-1.47)$ & $1.22(1.08-1.38)$ & $916(26.80)$ & $1.22(1.08-1.37)$ & $1.14(1.00-1.30)$ \\
\hline & $\mathrm{T} 2$ & $869(25.42)$ & Reference & Reference & $793(23.19)$ & Reference & Reference \\
\hline \multirow{2}{*}{ Calcium } & $\mathrm{T} 2$ & $907(25.90)$ & Reference & Reference & $853(24.36)$ & Reference & Reference \\
\hline & T3 & 895 (26.35) & $1.04(0.93-1.17)$ & $1.11(0.98-1.26)$ & 767 (22.58) & $0.91(0.81-1.03)$ & $0.97(0.85-1.10)$ \\
\hline
\end{tabular}

${ }^{*}$ Adjusted for age, gender, body mass index, history of hypertension, diabetes mellitus, dyslipidemia, stroke or TIA, atrial fibrillation/flutter, peripheral vascular disease, heart failure, stroke type, TOAST, current smoke, cholesterol-lowering agents, antihypertensive agents, hypoglycemic agents, antiplatelet agents, anticoagulant agents, NIHSS, total cholesterol, high-density cholesterol lipoprotein, fasting blood glucose, estimated glomerular filtration rate, and high sensitivity C-reactive protein. mRS, modified Rankin Scale. 